\title{
Irreversible Electroporation
}

\author{
Govindarajan Narayanan, $\mathrm{MD}^{1}$ \\ ${ }^{1}$ Division of Vascular and Interventional Radiology, Department of \\ Radiology, University of Miami-Miller School of Medicine, Miami, \\ Florida
}

Semin Intervent Radiol 2015;32:349-355

\begin{abstract}
Address for correspondence Govindarajan Narayanan, MD, Division of Vascular and Interventional Radiology, Department of Radiology, University of Miami-Miller School of Medicine, 1476 NW 12th Avenue, Miami, FL 33136 (e-mail: gnarayanan@med.miami.edu).
\end{abstract}
Abstract
Keywords
- irreversible electroporation
- ablation
- interventional radiology
- liver
- pancreas
- prostate

Irreversible electroporation (IRE) is a predominantly nonthermal ablative technology that uses high-voltage, low-energy DC current pulses to induce cell death. Thermal ablative technologies such as radiofrequency ablation, microwave ablation, and cryoablation have several applications in oncology but have limitations that have been established. IRE has shown promise to overcome some of these limitations. This article reviews the basics of the technology, patient selection, clinical applications, practical pointers, and the published data.
Objectives: Upon completion of this article, the reader will be able to identify the important technical considerations, patient selection, and outcomes associated with irreversible electroporation.

Accreditation: This activity has been planned and implemented in accordance with the Essential Areas and Policies of the Accreditation Council for Continuing Medical Education (ACCME) through the joint providership of Tufts University School of Medicine (TUSM) and Thieme Medical Publishers, New York. TUSM is accredited by the ACCME to provide continuing medical education for physicians.

Credit: Tufts University School of Medicine designates this journal-based CME activity for a maximum of 1 AMA PRA Category 1 Credit $^{\mathrm{TM}}$. Physicians should claim only the credit commensurate with the extent of their participation in the activity.

Irreversible electroporation (IRE) is a technology that uses DC current up to $3 \mathrm{kV}$ to induce cell death. Application of an electric field across a cell leads to formation of nanopores, a process called poration. When the voltage applied is up to $1 \mathrm{kV}$ the phenomenon is termed reversible electroporation, a technology that has been around for a few decades and has several applications in biotechnology and medicine. Tempo- rary nanopores in the cell membrane allow introduction of genes and drugs into cells, which normally cannot penetrate the membrane. ${ }^{1}$

Davalos, Mir, and Rubinsky demonstrated that IRE can be used as an independent modality for ablation, confirming it in studies of cells, ${ }^{2}$ small animal models, ${ }^{3}$ and liver of large animal models. ${ }^{4}$ Their work highlighted some of the unique characteristics of IRE, such as sparing blood vessels and bile ducts, rapid activation of the immune system, and the potential to treat tumors near blood vessels.

Lee et $\mathrm{al}^{5}$ studied the effectiveness of IRE in liver tissue and radiologic-pathologic correlation of IRE-induced cell death in Yorkshire pigs. In this model, bile ducts and vessel integrity were proven to be preserved and treatment areas were sharply demarcated.

\section{NanoKnife System}

IRE technology is commercially available as the NanoKnife, manufactured and sold by AngioDynamics (Latham, NY). This device has a 510k clearance from the Food and Drug Administration (FDA) for soft-tissue ablation; the use of the NanoKnife in organs is considered off label. The device has three components:
Issue Theme New Tools, Techniques, and Technologies; Guest Editor, Hector Ferral, MD
Copyright (c) 2015 by Thieme Medical Publishers, Inc., 333 Seventh Avenue, New York, NY 10001, USA. Tel: +1(212) 584-4662.
DOI http://dx.doi.org/ 10.1055/s-0035-1564706. ISSN 0739-9529. 
the generator, monopolar probes, and the AccuSync device (-Fig. 1).

The generator delivers low-voltage, high-energy DC current through the active tips of monopolar probes connected to the generator. A minimum of two monopolar probes are required to create a treatment zone, and depending on the size of the lesion, a maximum of six probes can be used, which can be used in odd or even number configurations. The energy is delivered between two probes at a time. Treatment planning is performed using the software incorporated in the system with a touch screen interface. The software helps determine the number of probes required to create the desired ablation volume.
The monopolar probes are 19-gauge needles with $1 \mathrm{~cm}$ depth markings along the shaft of the probe. They are available in 15 and $20 \mathrm{~cm}$ lengths. The active tip can be exposed between 1 and $4 \mathrm{~cm}$, depending on the desired size of the ablation zone and the depth of the lesion. The exposed electrode surface is echogenic for improved visibility when using ultrasound guidance for placement. The probes are inserted parallel to each other; it is important to avoid convergence or divergence of probes, which can result in a nonuniform ablation zone. Spacing between the probes is critical, with the ideal spacing being between 1.5 and $2 \mathrm{~cm}$ for the largest ablation volume. Spacing more than the recommended distance increases the chances of high current errors.
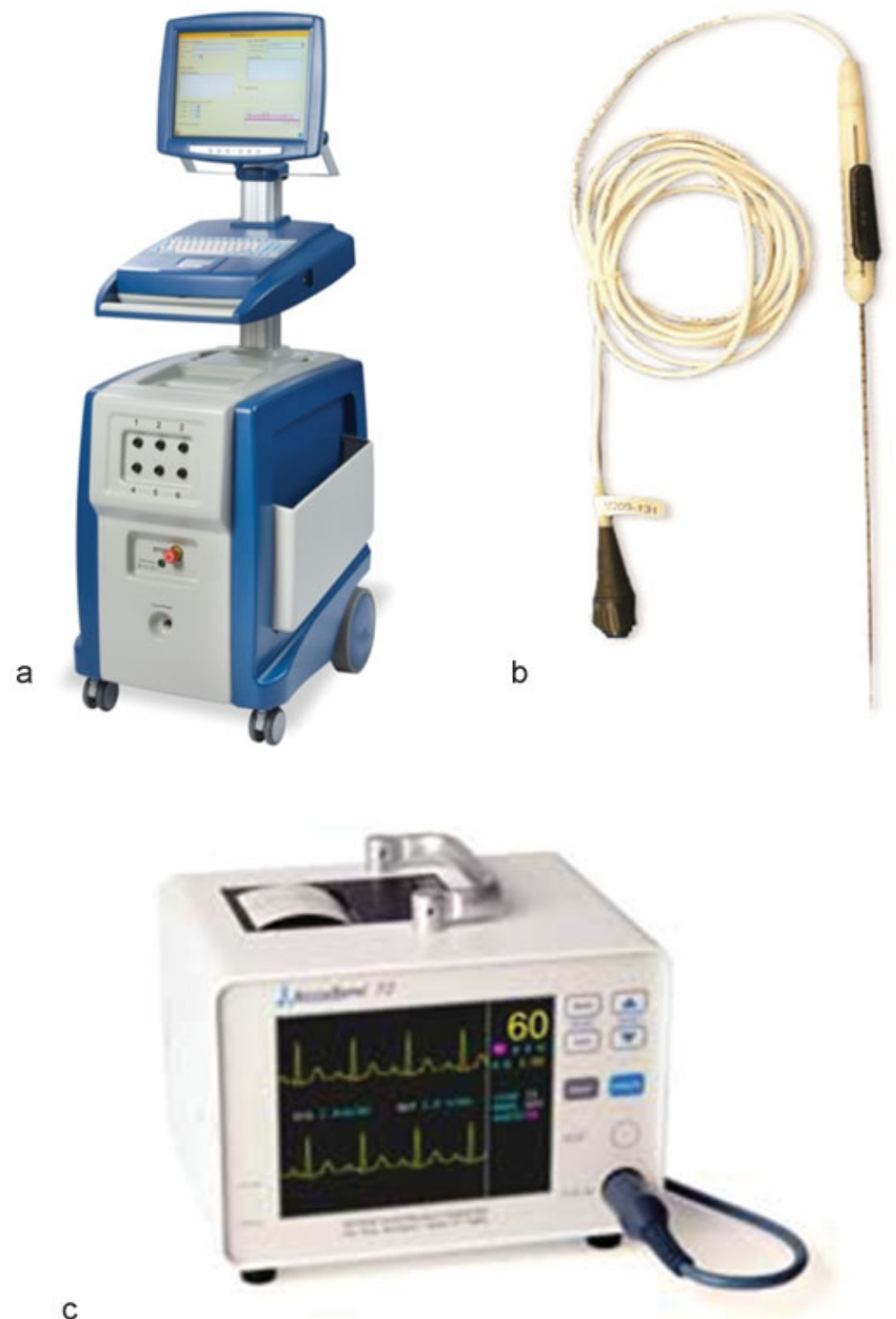

Fig. 1 (a-c) Components of the NanoKnife device (AngioDynamics, Latham, NY): the generator, monopolar probes, and the AccuSync device. 
The AccuSync 72 is a gating device, which is a standard part of the package of the NanoKnife system after four patients experienced transient ventricular arrhythmia in the first human experience with IRE. ${ }^{6}$ The AccuSync 72 is a five-lead system and a printer, which synchronizes pulse delivery with the patient's electrocardiogram (ECG). When an electrical energy pulse is delivered, the AccuSync system detects the rising slope of the R-wave and sends a signal to the NanoKnife generator. There is 0.05 second delay before the generator delivers the pulse in the refractory period of the cardiac cycle, which reduces the risk of a cardiac event (-Fig. 2).

\section{Patient Selection}

Patients should be reviewed in a multidisciplinary tumor board, and if determined to be candidates for IRE they are then evaluated in the interventional oncology clinic to complete the preprocedure workup. All patients are informed about the off-label use of the technology. Performance status is documented using the Eastern Cooperative Oncology Group (ECOG) criteria. A detailed cardiac history is obtained and coagulation tests, renal function, metabolic panel, and serum blood count are evaluated. Preprocedure cross-sectional imaging and positron emission tomography/computed tomography (PET/CT) (as indicated) should be obtained within 1 month of the consultation. A consult with the anesthesia service is also required to obtain clearance for general anesthesia.

\section{Contraindications}

A history of cardiac arrhythmias is a contraindication for IRE, as the electrical pulses cannot by synchronized with the cardiac R-waves, thereby increasing the risk for ventricular arrhythmias. Other contraindications include a pacemaker-dependent patient and unsafe access rates for a percutaneous approach (such as overlying colon obscuring the window or varices in the path to the lesion). Varices commonly seen in pancreatic cancer patients can bleed profusely. Prescreening imaging should be evaluated to identify this risk.

\section{Percutaneous Technique}

IRE can be performed with CT or ultrasound guidance under general anesthesia. Defibrillator pads are placed prior to initiating the procedure to intervene in case of a ventricular arrhythmia. General anesthesia is mandatory for IRE procedures; the procedure cannot be performed under conscious sedation. The patient is intubated and vital signs are constantly monitored throughout the procedure by the anesthetist. Complete neuromuscular blockade is essential to counteract the stimulation of skeletal muscle due to the high voltage delivered and to prevent movement of probes secondary to muscle contraction. Commonly used paralyzing agents include cisatracurium besylate (Nimbex; Abbott Laboratories, Chicago, IL) or rocuronium bromide (Hospira, Inc., Lake Forest, IL).

A triple-phase CT of the abdomen is performed with administration of $65 \mathrm{~mL}$ of iodinated contrast followed by a $65-\mathrm{mL}$ saline bolus, and the intended configuration and number of probes are determined.

Following placement of the probes with imaging guidance, a three-dimensional (3D) CT scan image is obtained to confirm the position of the probes and the distance between each pair. A twitch monitor is used to check the number of twitches, which should be between 0 and 1 . This is done to confirm adequate muscle relaxation before initiation of treatment. The twitch monitor is then turned off and treatment is initiated.

A total of 70 high-voltage DC current pulses $(1,500-3,000$ $\mathrm{V})$ are delivered between the probes to complete the treatment between one probe pair. Depending on the number of pairs, the generator automatically switches to the next pair(s) to complete the treatment. The treatment time between a pair is 70 seconds. A postprocedure CT scan is obtained to assess for any immediate complications. Contrast is used in patients with intact renal function to evaluate vascularity.

\section{Postprocedure Care}

Following IRE, all patients are admitted for overnight observation. A Dilaudid patient-controlled analgesia pump is provided for pain control. Postprocedure hematocrit level and chemistry panel are evaluated prior to discharge.

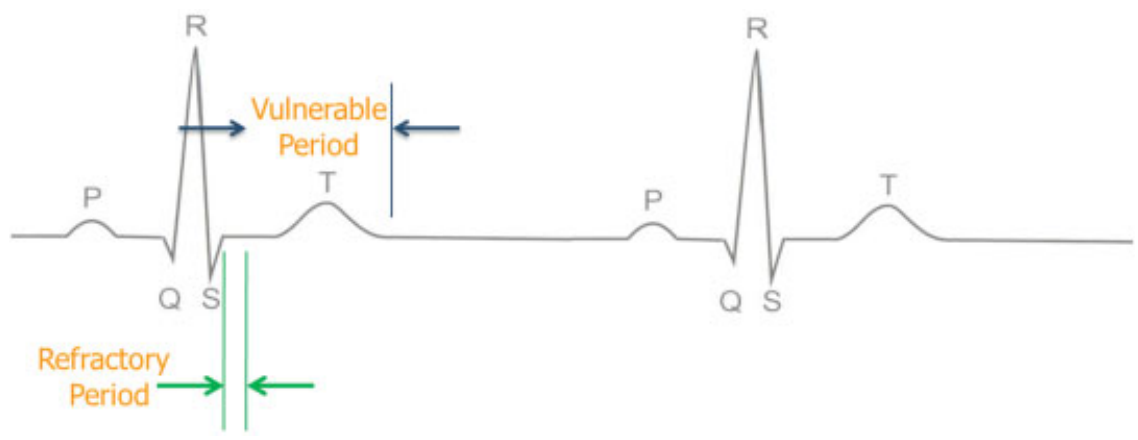

Fig. 2 Sync device (e.g., AccuSync 72) senses the rising slope of the R-wave, and sends a signal to the NanoKnife (AngioDynamics, Latham, NY). The NanoKnife waits 50 milliseconds ( 0.05 second) and delivers 1 energy pulse. The energy pulse is delivered during (or just before) the ventricular refractory period. 
Antibiotic coverage with metronidazole is continued for 3 days (500 mg, three times daily), and ciprofloxacin is continued for 5 days ( $500 \mathrm{mg}$, two times daily). Ondansetron (4 mg) is prescribed as needed to alleviate nausea.

\section{Clinical Applications}

\section{Liver}

The first study that established the safety of IRE in humans was published by Thomson et al. ${ }^{6}$ Since then, several publications have documented the safety and efficacy of IRE as an ablative tool.

In liver lesions close to vasculature, bile ducts and adjacent structures pose a challenge when thermal ablative techniques are used. The heat-sink effect is a limitation of percutaneous radiofrequency ablation, where blood flow in a vessel close to the ablation zone causes loss of heat secondary to convection; this leads to incomplete ablation. In addition, lesions in close proximity to biliary ducts, and the porta-hepatis, carry a risk of collateral injury with thermal ablation.

Kingham et al studied the safety and short-term outcomes of IRE in ablating perivascular malignant liver tumors. These authors published a retrospective review of 28 patients who had 65 tumors treated with IRE via either an open approach or percutaneously. ${ }^{7}$ These results demonstrated the safety of treating perivascular malignant hepatic tumors with IRE.

The effect of percutaneous IRE ablations on peribiliary tumors was studied by Silk et al, following ablation of hepatic tumors located $<1 \mathrm{~cm}$ from major bile ducts. ${ }^{8}$ Eleven patients had 22 hepatic metastases treated with IRE in 15 sessions, with at least one tumor within $1 \mathrm{~cm}$ of the common, left, or right hepatic duct. Median size of the treated tumor was $3.0 \mathrm{~cm}$ (mean, $2.8 \mathrm{~cm} \pm 1.2$; range, $1.0-4.7 \mathrm{~cm}$ ). This study concluded that IRE may be a treatment option for centrally located liver tumors with margins adjacent to major bile ducts where thermal ablation techniques are contraindicated.

A retrospective review on the effects of IRE on vasculature in close proximity to the ablation zone included a total of 129 lesions that were treated with percutaneous IRE for primary and metastatic tumors in different organs. ${ }^{9} \mathrm{~A}$ total of 158 vessels in close proximity to the ablation zone were examined for caliber, patency, and flow defects on follow-up contrastenhanced CT and magnetic resonance imaging (MRI) scans. Close proximity was defined as vessels within 0 to $1 \mathrm{~cm}$ from the treatment zone. Overall mean follow-up was 10.3 months. Abnormal vascular changes were noted in 7 of $158(4.4 \%)$ vessels. These data from 101 patients demonstrated the safety of IRE in the treatment of tumors near large blood vessels and tumors already encasing vessels (-Fig. $\mathbf{3}$ ).
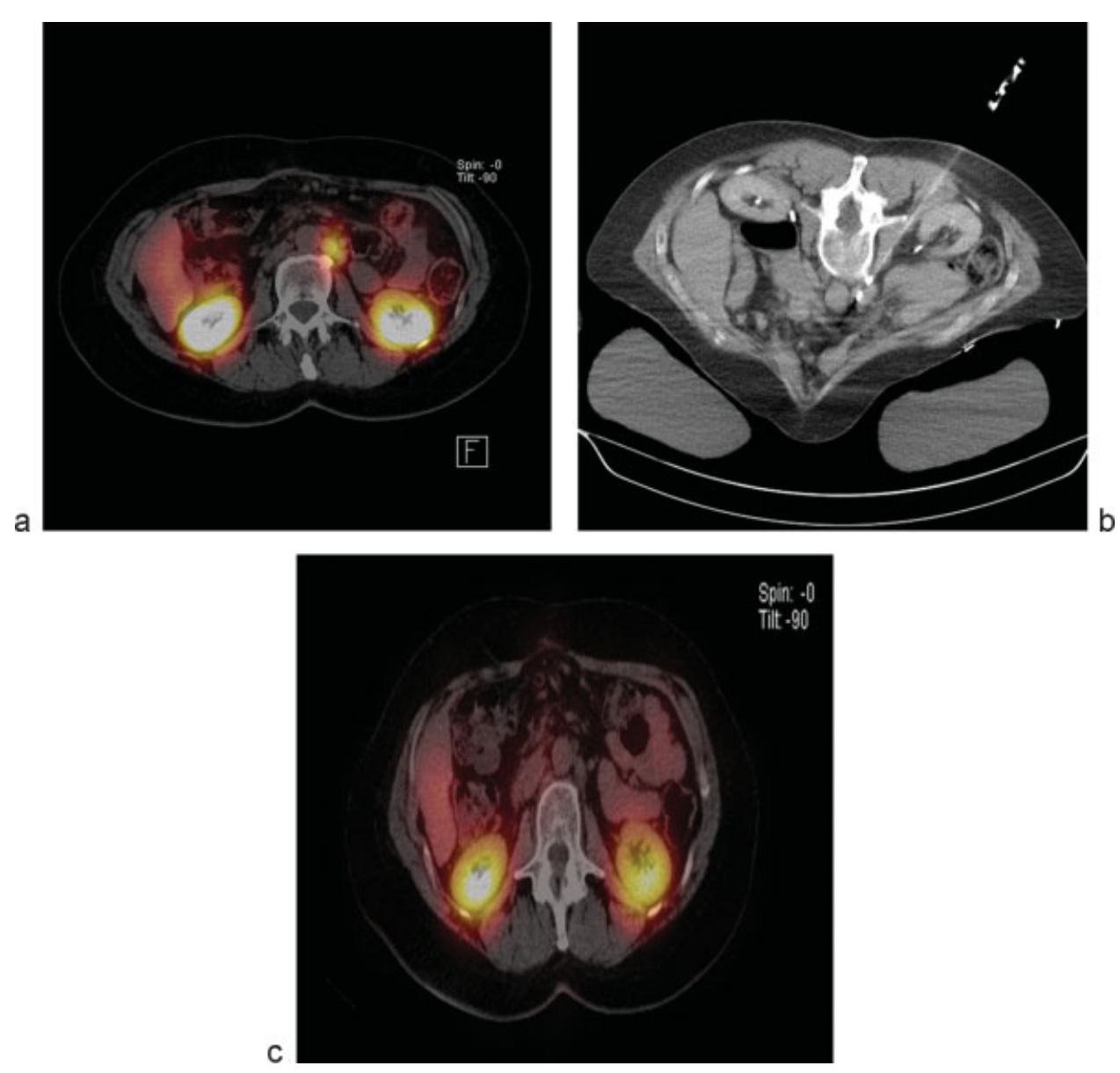

Fig. 3 Octreotide scan (a) demonstrates a metastatic foci of neuroendocrine tumor in the periaortic location. The lesion was treated with irreversible electroporation (b) and follow-up imaging demonstrates resolution of the focus (c). 
Scheffer et al studied the pathological response of colorectal liver metastases (CRLM) treated with IRE in a safety and feasibility trial. ${ }^{10}$ Ten patients with resectable CRLM underwent laparotomy, and 10 lesions with a mean diameter of $2.4 \mathrm{~cm}$ were successfully electroporated and resected. These lesions took on average 84 minutes later (range, 51-153 minutes) for treatment. Safety and feasibility were assessed based on adverse events, laboratory values, technical success, and intraoperative ultrasound findings. Tissue response was assessed using Triphenyl tetrazolium chloride (TTC) vitality staining and (immuno) histochemical staining (hematoxylin and eosin [HE], complement 3D, and caspase-3). One minor transient cardiac arrhythmia occurred during IRE. Ultrasound typically showed a sharply demarcated hypoechoic ablation zone around the tumor. TTC showed avitality of all lesions, covering the complete tumor in 8/10 lesions. Although immunohistochemistry proved heterogeneous and difficult to interpret within the tumors, it confirmed irreversible cell damage in the tumor-free margin of all specimens and that the IRE-induced cell death occurred in CRLM within 1 hour. The ablation zone showed a sharp demarcation between avital and vital tissue, and apoptosis was noted to be involved in cell death of colorectal liver metastases after IRE (-Fig. 4).

\section{Prostate}

Given its ability to spare vasculature and nerves, there is considerable interest in the role of IRE in the prostate gland. Effects of IRE in prostate ablation were initially studied in animals by Onik et al. ${ }^{11}$ IRE was used to treat the prostate of six male dogs. In one of the dogs, the lesions were made purposely to include the rectum, urethra, and neurovascular bundle. Subjects were followed up for 1 to 14 days before sacrifice. The margins of the IRE lesions were very distinct with a narrow zone of transition from normal to complete necrosis. There was complete destruction within the IRE lesion, and rapid resolution of the lesions with marked shrinkage within 2 weeks. Structures such as the urethra, vessels, nerves, and rectum were unaffected by the IRE application.

Valerio et al evaluated the safety and clinical feasibility of focal IRE of the prostate. ${ }^{12}$ Thirty-four patients were treated with IRE, delivered under transrectal ultrasound guidance with two to six IRE probes, and positioned transperineally within the cancerous lesion. Complications were
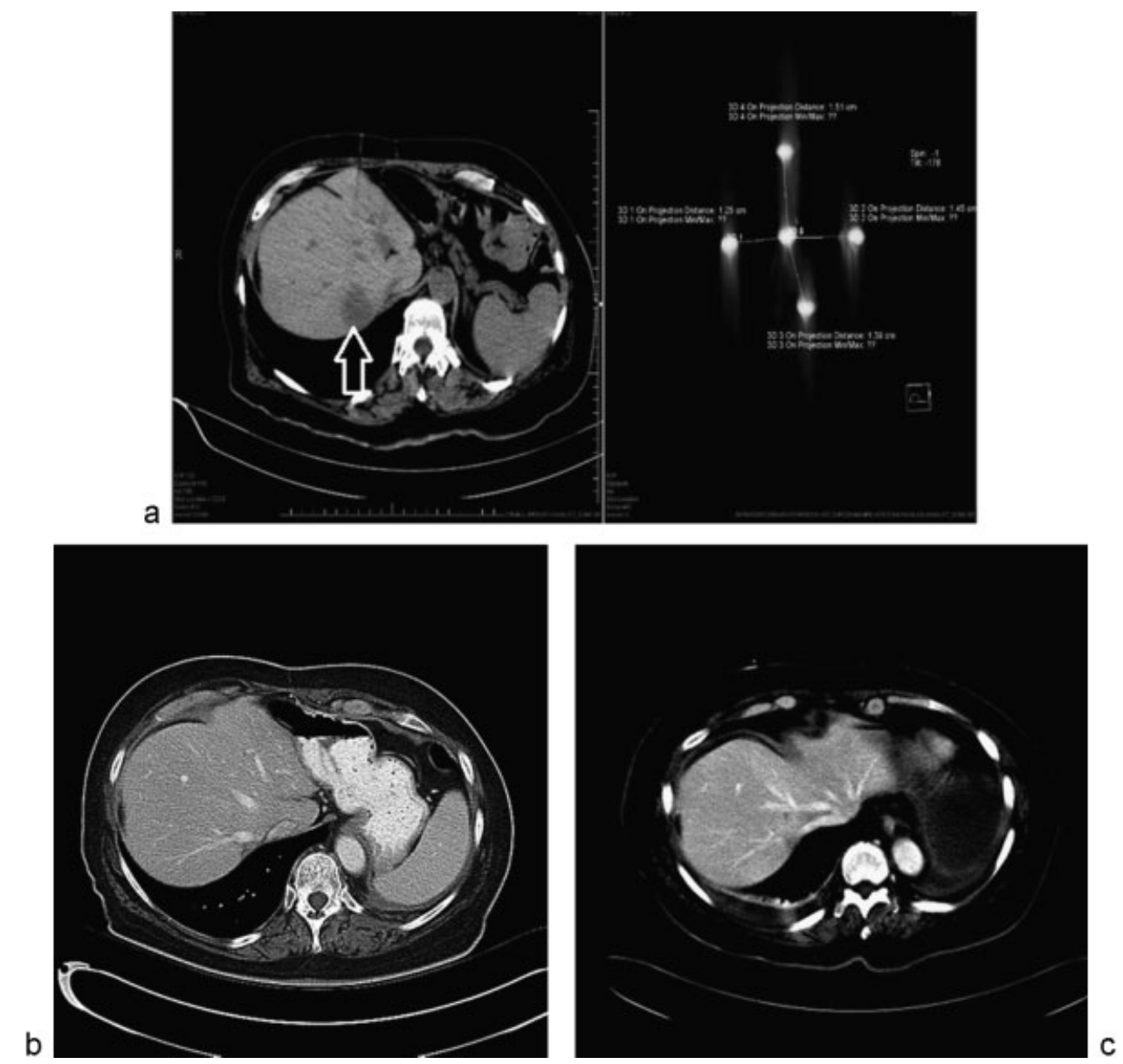

Fig. 4 Single metastatic focus in a lung cancer patient treated with irreversible electroporation. (a) CT scan demonstrating the metastatic focus in the liver (arrow), and placement of the five probes used to treat the lesion. (b and c) Follow-up CT scan performed 8 and 12 months posttreatment, respectively, demonstrating no evidence for recurrent disease. 
recorded, and the functional outcome was physician reported in all patients with at least 6 months follow-up. A contrast-enhanced MRI 1 week after the procedure was performed to assess treatment effect, with a further MRI at 6 months to rule out evidence of residual visible cancer. Overall, 34 patients with a mean age of 65 years (standard deviation $= \pm 6$ ) and a median prostate-specific antigen (PSA) of $6.1 \mathrm{ng} / \mathrm{mL}(-1)$ (interquartile range [IQR] = 4.37.7) were included. After a median follow-up of 6 months (range, 1-24), 12 grade 1 and 10 grade 2 complications occurred; no patient had grade 3 or higher complications. From a functional point of view, 100\% (24/24) of the patients were continent, and potency was preserved in 95\% (19/20) men who were potent before treatment. The median PSA after 6 months of $3.4 \mathrm{ng} / \mathrm{mL}$ (interquartile range $=1.9-4.8 \mathrm{ng} / \mathrm{mL}$ was noted. MRI showed suspicious residual disease in six patients, of whom four underwent another form of local treatment. Focal IRE in the prostate holds promise with a low toxicity profile and encouraging genitourinary functional outcomes.

\section{Pancreas}

Pancreatic cancer is currently the fourth leading cause of cancer death in men and women. Most patients who present with pancreatic cancer are not surgical candidates at diagnosis, as $40 \%$ of patients present with Stage 3 locally advanced pancreatic cancer (LAPC) and $40 \%$ present with metastatic pancreatic cancer (MPC). ${ }^{13-15}$ The 1 - and 5-year relative survival rates are poor (27 and $6 \%$ for all stages combined, respectively). ${ }^{13}$ In patients with local disease, 5-year survival is $24 \%$, compared with those with distant disease in which it is only $2 \% .{ }^{13}$ Thermal ablation in the pancreas has been in the surgical domain, and different series have reported significant morbidity and mortality. ${ }^{16,17}$

Open, laparoscopic, approaches have been studied and initial evidence shows an acceptable safety profile with a possible survival benefit in selected patients. ${ }^{18}$

The introduction of the percutaneous technique has provided a minimally invasive option for select patients with pancreatic cancer. Ideal patients for percutaneous IRE of the pancreas should have an ECOG status of 0 to 1 , with Stage 3 LAPC or low volume Stage 4 metastatic disease that has been stable over time. In patients with borderline resectable or unresectable LAPC, IRE may downsize tumor to allow resection. ${ }^{19}$ In patients who have undergone resection, local recurrence may also be managed with IRE. Patients with an incompetent ampulla from a stent or resection from a Whipple procedure are at increased risk of infection and require extended antibiotic coverage.

All patients receive bowel preparation similar to a colonoscopy to decrease risk of infection, and to decrease the chance of colon obscuring the pancreatic bed. Placement of a nasogastric tube allows administration of contrast to delineate small bowel and permits insufflation of the stomach to push the colon caudally, if necessary.

Pancreatitis, vascular injury and bleeding following probe placement, collateral injury to adjacent structure, and infection are possible complications following IRE. If pancreatitis does occur, conservative management is indicated and includes pain control, keeping the nasogastric tube to low intermittent suction, intravenous hydration, and nothing by mouth until amylase and lipase are normal.

Early data on the use of IRE in the management of pancreatic cancer holds significant promise. IRE has been used in the operating room for the treatment and margin accentuation of borderline and locally advanced pancreatic cancer, and IRE using an open surgical approach in LAPC patients has been shown to potentially improve overall survival. $^{18}$

Narayanan et al reported a retrospective review of 43 patients who underwent 50 IRE procedures using a percutaneous approach. ${ }^{19}$ ( - Fig. 4). Thirty out of 43 (69.8\%) patients had LAPC, and the overall survival in these patients was 16.2 months (95\% CI: 10.1-22.3 months). The remaining 13 patients (30.2\%) had MPC, and had an overall survival of 8.6 months (95\% CI: 3.1-14.1 months). Nineteen patients (44\%) received chemotherapy after IRE. Two patients with LAPC were downstaged and were able to undergo surgery with R0 (margin negative) resections.

In this series, the complications from pancreatic IRE included abdominal pain $(n=10)$, pancreatitis $(n=7)$, hematoma $(n=7)$, spontaneous pneumothorax $(n=1)$, the need for a duodenal stent placement $(n=1)$, main portal vein thrombosis (found at 1 month follow-up CT scan) $(n=1)$, and sepsis 48 hours post-IRE $(n=1)$. Cases of pancreatitis were managed with an extended hospital stay and conservative management. Out of the 20 patients who had expired at the time of publication, no deaths were directly related to the procedure. This series demonstrated the potential survival benefit in selected patients with localized or metastatic pancreatic cancer using percutaneous IRE.

Currently, the PANFIRE study, a phase I clinical trial designed to investigate the safety, feasibility, and efficacy of percutaneous IRE in patients with LAPC, has completed recruitment. ${ }^{20}$ Phase II/III randomized controlled trials to help define the role of IRE in the treatment algorithm of pancreatic cancer are being proposed and will help define the role of IRE in the treatment algorithm of patients with pancreatic cancer.

\section{Conclusion}

In a little over 5 years since IRE has been commercially available, it has moved from being a novelty to a technology that has demonstrated the potential to be a valuable addition to the other ablation tools. Evidence from surgical and interventional radiology literature shows promise for interventions in areas such as prostate and pancreatic cancer and other niche applications such as the ability to treat near vasculature.

\section{References}

1 Rubinsky B. Irreversible electroporation in medicine. Technol Cancer Res Treat 2007;6(4):255-260 
2 Miller L, Leor J, Rubinsky B. Cancer cells ablation with irreversible electroporation. Technol Cancer Res Treat 2005;4(6):699-705

3 Edd JF, Horowitz L, Davalos RV, Mir LM, Rubinsky B. In vivo results of a new focal tissue ablation technique: irreversible electroporation. IEEE Trans Biomed Eng 2006;53(7):1409-1415

4 Rubinsky B, Onik G, Mikus P. Irreversible electroporation: a new ablation modality-clinical implications. Technol Cancer Res Treat 2007;6(1):37-48

5 Lee EW, Chen C, Prieto VE, Dry SM, Loh CT, Kee ST. Advanced hepatic ablation technique for creating complete cell death: irreversible electroporation. Radiology 2010;255(2):426-433

6 Thomson KR, Cheung W, Ellis SJ, et al. Investigation of the safety of irreversible electroporation in humans. J Vasc Interv Radiol 2011; 22(5):611-621

7 Kingham TP, Karkar AM, D’Angelica MI, et al. Ablation of perivascular hepatic malignant tumors with irreversible electroporation. J Am Coll Surg 2012;215(3):379-387

8 Silk MT, Wimmer T, Lee KS, et al. Percutaneous ablation of peribiliary tumors with irreversible electroporation. J Vasc Interv Radiol 2014;25(1):112-118

9 Narayanan G, Bhatia S, Echenique A, Suthar R, Barbery K, Yrizarry J. Vessel patency post irreversible electroporation. Cardiovasc Intervent Radiol 2014;37(6):1523-1529

10 Scheffer HJ, Nielsen K, van Tilborg AA, et al. Ablation of colorectal liver metastases by irreversible electroporation: results of the COLDFIRE-I ablate-and-resect study. Eur Radiol 2014;24(10):2467-2475

11 Onik G, Mikus P, Rubinsky B. Irreversible electroporation: implications for prostate ablation. Technol Cancer Res Treat 2007;6(4): 295-300
12 Valerio M, Stricker PD, Ahmed HU, et al. Initial assessment of safety and clinical feasibility of irreversible electroporation in the focal treatment of prostate cancer. Prostate Cancer Prostatic Dis 2014; 17(4):343-347

13 American Cancer Society. Cancer Facts \& Figures 2014. American Cancer Society 2014. Available at: http://www.cancer.org/acs/ groups/content/@research/documents/webcontent/acspc042151.pdf. Accessed July 2015

14 Jemal A, Bray F, Center MM, Ferlay J, Ward E, Forman D. Global cancer statistics. CA Cancer J Clin 2011;61(2):69-90

15 Ghosn M, Kourie HR, El Karak F, Hanna C, Antoun J, Nasr D. Optimum chemotherapy in the management of metastatic pancreatic cancer. World J Gastroenterol 2014;20(9): $2352-2357$

16 Girelli R, Frigerio I, Salvia R, Barbi E, Tinazzi Martini P, Bassi C. Feasibility and safety of radiofrequency ablation for locally advanced pancreatic cancer. Br J Surg 2010;97(2):220-225

$17 \mathrm{Wu} \mathrm{Y}$, Tang Z, Fang H, et al. High operative risk of cool-tip radiofrequency ablation for unresectable pancreatic head cancer. J Surg Oncol 2006;94(5):392-395

18 Martin RC II, McFarland K, Ellis S, Velanovich V. Irreversible electroporation in locally advanced pancreatic cancer: potential improved overall survival. Ann Surg Oncol 2013;20(Suppl 3): S443-S449

19 Narayanan G, Froud T, Suthar R, Barbery K, Yrizarry J. Percutaneous irreversible electroporation (IRE) in the management of pancreatic cancer. J Vasc Interv Radiol 2014;25(3):S35

20 Meijerink M. The PANFIRE study. Available at: https://clinicaltrials.gov/ct2/show/NCT01939665. Accessed July 2015 